(Aus dem Bakteriologischen Institut des Allgemeinen Krankenhauses Hamburg-Altona. - Prof. Dr. Zeissler.)

\title{
Berichtigung \\ zu Dr. Alfred Primnigs Arbeit: ,Uber die Beziehungen des Gasbrandbacillus zur Melaena neonatorum.6
}

(Diese Zeitschrift Bd. 61, S. 626.)

Von

\section{J. Zeissler.}

In seiner unter obigem Titel erschienenen Arbeit behauptet Primnig „Neben dem toxinbildenden pathogenen Typus des Fraenkel-Welchschen Bacillus gibt es einen weit häufiger vorkommenden saprophytären Typus, von dem $J$.Zeissler sagt, daß er der ubiquitärste und vielleicht weitverbreiteste Keim der Erde sei".

Diese Darstellung könnte den Anschein erwecken, als ob ich selbst sie für richtig hielte oder gar die wissenschaftlichen Voraussetzungen für die geschaffon hätte.

Das Gegenteil ist der Fall. Trotz praktischer Bearbeitung von vielen Hunderten verschiedener Stämme des Fraenkelschen Gasbacillus (B. Welchii, B. perfringens) in meinem Institut ist es hier bis jetzt nicht gelungen, einen Unterschied zwischen aus Erde oder aus pathogenem menschlichen Material gewonnenen Keimen dieser Art festzustellen. Insbesondere hat sich bis jetzt auch kein aus Erde gewonnener Gasbacillus bei der hier geübten Behandlung als apathogen für Meerschweinchen gezeigt. Diese unsere Feststellungen stehen in bestem Einklang mit den Erfahrungen der Kriegs- und Unfallchirurgie, die uns Iehren, daß erdbeschmutzte Wunden in hohen Maße gasbrandgefährdet sind, und mit der Tatsache, daß es leicht gelingt, bei geeigneten Versuchs. tieren durch Verimpfung von Erde Gasbrand experimentell zu erzeugen.

Seine von diesen ganz allgemein bekannten Tatsachen so ganz abweichende Anschauung hätte Primnig sehr gründlich belegen müssen. Nicht nur durch den Bericht über einen einzigen selbst beobachteten Krankheitsfall, der über die Einheitlichkeit der Bacillenart Fraenkelscher Gasbacillus (B. Welchii, B. perfringens) an sich kein Urteil erlaubt, und eine Reihe von Zitaten aus der Literatur, die zum Teil ihrerseits sich wiederum nur auf Zitate stützen.

Mit der - wie hier ausgeführt - vollkommen unbegründeten und außerdem mit den Erfahrungen des praktischen Lebens in Widerspruch stehenden Behauptung von ,verschiedenen Typen" (pathogenen und 
apathogenen) des Fraenkelschen Gasbacillus (B. Welchii, B. perfringens) fallen auch die von Primnig auf sie gegründeten Folgerungen.

\section{Schriftennachweis.}

Primnig, Alfred: Z. Kinderheilk. 61, 626 (1940). - Zeissler, J.: Die Gasödeminfektionen des Menschen. Handbuch der pathogenen Mikroorganismen von Kolle, Kraus, Uhlenhuth, Bd. IV. Jena, Berlin u. Wien: Gustav Fischer 1928. Zbl. Chir. 66, 1013 (1939). - Zeissler u. Raßfeld: Die anaerobe Sporenflora der Europäischen Kriegsschauplätze 1917. Jena: Gustav Fischer 1928.

Bakteriologisches Institut des Allgemeinen Krankenhauses Hamburg-Altona. 